\title{
Prevalencia del cáncer de cabeza y cuello en el Hospital de Especialidades Eugenio Espejo en el periodo 2002-2015 en Quito, Ecuador
}

\author{
Mayra E. Paltas-Miranda ${ }^{1 *}$, Ahmad W. Mushtaq-Wali ${ }^{2}$ y Ma. Gabriela Haye-Biazevic ${ }^{3}$ \\ ${ }^{1}$ Facultad de Odontología, Universidad Central del Ecuador, Quito, Ecuador; ${ }^{2}$ Servicio de Oncología, Hospital Eugenio Espejo, Quito, Ecuador; \\ ${ }^{3}$ Facultad de Odontología, Universidad de São Paulo, São Paulo, Brasil
}

\section{Resumen}

Objetivo: Determinar la prevalencia de cáncer de cabeza y cuello en el Servicio de Oncología del Hospital de Especialidades Eugenio Espejo, periodo 2002 al 2015. Métodos: Es un estudio epidemiológico, analítico y transversal; los datos fueron tomados de las historias clínicas de pacientes atendidos con cáncer de cabeza y cuello, fueron registrados en una planilla de Excel, codificados y analizados en el paquete de estadística STATA versión 14.0, estadística descriptiva, los test de Chi cuadrada y de Poisson, con un nivel de significancia $0.05 \%$ y una confiabilidad del 95\%. Resultados: Existió una prevalencia discreta en sexo femenino con el 50.7\%, la edad de 50-60 años el 20.3\%, nivel de educación (primaria) el 53.1\%, residencia «sierra» el 76.7\%, ocupación quehaceres domésticos el 38.4\%; la región anatómica cavidad bucal (36.4\%); el tratamiento quirúrgico fue el 38.9\%; tiempo de tratamiento hospitalario (de 1 a 180 días) el 44.5\% y el 31.8\% finalizó el tratamiento. Conclusiones: El cáncer de cabeza y cuello prevaleció más en el sexo femenino entre la edad de 55 años \pm 5 años. La mayoría era de la región sierra, con bajo nivel de instrucción, y realizaban quehaceres domésticos. La cavidad bucal fue la región anatómica más afectada.

Palabras clave: Cáncer. Cabeza y cuello. Tratamiento. Epidemiología.

\section{Prevalence of head and neck cancer in the Eugenio Espejo Specialties Hospital, period 2002-2015, Quito, Ecuador}

\section{Abstract}

Objective: To determine the prevalence of head and neck cancer in the Oncology Service of the Eugenio Espejo Hospital, from 2002 to 2015. Methods: This is an epidemiological, analytical and cross-sectional study, the data was retrieved from the medical records of patients who were treated with head and neck cancer, all data was collected and transcribed onto an Excel spreadsheet, then coded and analyzed in STATA version 14.0. Descriptive Statistics and the Chi-square and Poisson tests were used with a level of significance of $0.05 \%$ at a level of confidence of $95 \%$. Results: There was a prevalence in female gender of $50.7 \%$, the age from 50 to 60 years represented $20.3 \%$, level of education (elementary) with $53.1 \%$, place of residence (highlands) with $76.7 \%$, occupation household chores with $38.4 \%$, anatomical region oral cavity $36.4 \%$, under surgical treatment $38.9 \%$, the time of hospital treatment from 1 to 180 days $44.5 \%$ and $31.8 \%$ of the patients finished the treatment. Conclusions: Head and neck cancer prevailed in the female gender in the age of $55, \pm 5$ years, most were from the Andean Mountain (Sierra) region with low level of instruction and whom perform household chores, The oral cavity was the most affected anatomical region.

Key words: Cancer. Head and neck. Treatment. Epidemiology.

\section{Correspondencia:}

*Mayra E. Paltas-Miranda

E-mail: mayelhy9@ hotmail.com

2565-005X/C 2021 Sociedad Mexicana de Oncología. Publicado por Permanyer. Este es un artículo open access bajo la licencia CC BY-NC-ND (http://creativecommons.org/licenses/by-nc-nd/4.0/).
Fecha de recepción: 15-04-2020

Fecha de aceptación: 28-03-2021
Disponible en internet: 08-06-2021

Gac Mex Oncol. 2021;20(2):52-61 www.gamo-smeo.com 


\section{Introducción}

El cáncer es un problema de salud pública que afecta a todo el mundo. Es una enfermedad caracterizada por la alteración o mutación de los genes reguladores normales, este fenómeno conduce al desarrollo de células cancerígenas con la capacidad de invadir y destruir tejidos adyacentes, así como producir metástasis hacia otros órganos ${ }^{1,2}$.

Los cánceres de cabeza y cuello pueden ser el resultado de la exposición a múltiples factores de riesgo ${ }^{3}$. Son lesiones con muchas diferencias en su incidencia, origen del tumor, forma clínica, evolución, tratamiento y pronóstico ${ }^{4,5}$. Tanto en la prevalencia, como en la incidencia y mortalidad varían considerablemente en las diferentes partes del mundo ${ }^{6,7}$. Los cánceres de cabeza y cuello pueden comprometer diferentes regiones anatómicas, como los labios, cavidad bucal, faringe, laringe, senos paranasales y glándulas salivales ${ }^{8,9}$.

De acuerdo con la Organización Mundial de la Salud (OMS), los cánceres de la cavidad bucal y orofaringe son las neoplasias más frecuentes de la cabeza y cue$\| 0^{8,10,11}$. El cáncer de cabeza y cuello presenta una incidencia muy importante en las dos últimas décadas, tornándose la sexta causa de muerte en el mundo 5,12,13, 650,000 nuevos casos diagnosticados cada año y 300,000 muertes por esta condición en el mismo periodo, representando el $5 \%$ del total de las neoplasias malignas $8,14,15$. Posee una incidencia dos veces mayor el sexo masculino en relación con el femenino; el tabaquismo solo o conjuntamente con alcohol es la causa principal del cáncer de cabeza y cuello; la edad mas afectada está en $60 \pm 5$ años ${ }^{16,17}$. El carcinoma espinocelular y sus variantes es el tipo histológico más común y la causa del $90 \%$ de todas las neoplasias de cabeza y cuello $6,9,18$.

En relación con la prevalencia del cáncer de cabeza y cuello en un periodo de cinco años de acuerdo con datos relatados por la GLOBOCAN 2012, los países en desarrollo representan el $62.2 \%$ y los países desarrollados el $37.7 \%{ }^{10,19}$. Existe mayor incidencia en Asia (57.6\%), seguida de Europa (20.3\%), América del Norte (8.4\%), América Latina y el Caribe (7.0\%), África (5.8\%) y Oceanía $(0.9 \%)$. La tasa de mortalidad es en Asia del 65.9\%, en Europa del $16.8 \%$, en África del $6.7 \%$, en América Latina y el Caribe del $6.4 \%$, en América del Norte del $3.7 \%$ y en Oceanía del $0.5 \%{ }^{10,20}$.

América Latina está caracterizada por una alta prevalencia e incidencia de cáncer de cabeza y cuello, con gran variación entre los países. De todos los casos nuevos en el mundo el $6.5 \%$ ocurren en América del Sur y Central ${ }^{21}$, los países con mayor incidencia de cáncer de cavidad bucal son Cuba, Brasil, Uruguay y Puerto Rico ${ }^{10,15}$.

Su alta incidencia del cáncer de cabeza y cuello puede darse ciertamente por mayor crecimiento y envejecimiento de la población, también por cambios de comportamiento en los estilos de vida como hábitos de consumo de tabaco y alcohol, mala alimentación, inactividad física y ciertos comportamientos sexuales en edad temprana ${ }^{20}$.

Los riesgos ocupacionales tienen un efecto acumulativo a lo largo del tiempo, dando solidez a datos epidemiológicos de mayor prevalencia en pacientes sobre los 50 años, lo cual hace que muchos autores afirmen que la edad es el principal factor de riesgo para el desarrollo del cáncer, especialmente en la cavidad bucal $^{22}$. La exposición prolongada a la radiación ultravioleta y algunos productos químicos acompañados de la susceptibilidad genética individual son factores de alto riesgo para el desarrollo del cáncer en la región aerodigestiva superior'.

Las manifestaciones clínicas del cáncer de cabeza y cuello son muy variadas y dependen principalmente de: la localización del tumor, del tamaño de la masa tumoral primaria, la presencia o no de nódulos y linfáticos e invasión de estructuras adyacentes; pueden presentarse parestesias, dolor, hemorragia, úlceras, dolor de garganta, disfagia y pérdida de peso $^{4,14}$, que provocan graves consecuencias en la salud de los pacientes y pueden llevar a grandes mutilaciones, deformidades físicas y funcionales que afectan gravemente en el ámbito psicológico, económico, social, familiar y generalmente la calidad de vida de los pacientes que padecen esta enfermedad ${ }^{23}$.

El tratamiento debe ser multidisciplinario e incluye personal médico, oncólogo, fisioterapeuta, psicólogo, maxilofacial e inclusive la ayuda de los familiares. El tipo de tratamiento dependerá del estadio tumoral, de la localización anatómica y tamaño del tumor y si hay o no metástasis, procurando siempre mantener la funcionalidad de los órganos; el tratamiento quirúrgico y radioterapia son los procedimientos convencionales más utilizados para los cánceres de cabeza y cuello 4,9,17.

Actualmente, existen datos estadísticos sobre cáncer de cabeza y cuello del 2006 al 2010, relatados por el Registro Nacional de Tumores SOLCA Quito y datos relatados sobre cáncer en general por el Instituto Nacional de Estadísticas y Censos (INEC) ${ }^{24}$, mas hasta el momento no hay estudios de cáncer de cabeza y cuello realizados en hospitales estatales del 
Ecuador. Siendo el hospital de Especialidades Eugenio Espejo un hospital de referencia nacional, en el cual se atiende a un gran número de personas con nivel educacional y recursos económicos bajos, ello motivó para la realización de este estudio, cuyo objetivo es determinar la prevalencia del cáncer de cabeza y cuello de acuerdo con las condiciones demográficas, tipo y sitio anatómico del cáncer, tiempo y evolución del tratamiento.

\section{Métodos}

Se trata de un estudio epidemiológico, analítico y transversal. Este proyecto fue sometido al Comité de Bioética en Investigación y Docencia del Hospital de Especialidades Eugenio Espejo y al Comité de Ética en Investigación con seres humanos de la Facultad de Odontología de la Universidad de Sao Paulo FOUSP.

Todos los datos fueron obtenidos de las historias clínicas de pacientes con cáncer de cabeza y cuello, que fueron atendidos en el Servicio de Oncología del Hospital de Especialidades Eugenio Espejo, durante un periodo de 13 años, de enero del 2002 a diciembre del 2015. Una muestra de 211 historias clínicas fue considerada para esta investigación, que correspondía a todas las historias clínicas de pacientes con cáncer de cabeza y cuello diagnosticados por medio de examen clínico e histológico; se excluyeron todas las historias clínicas que no contaban con los datos necesarios para la investigación.

Se consideraron datos demográficos del paciente como edad, sexo, nivel de instrucción, lugar de residencia y profesión, datos relacionados con el tipo de cáncer de cabeza y cuello de acuerdo con la Clasificación Internacional de Enfermedades 10. ${ }^{\text {a }}$ revisión (CIE-10), datos relacionados con la localización anatómica del tumor, tipo de tratamiento recibido, tiempo de tratamiento hospitalario (para lo cual se consideraron las fechas de ingreso y egreso del hospital) y cuál fue la evolución del paciente con cáncer de cabeza y cuello (pacientes vivos que concluyeron el tratamiento, aquellos que murieron durante el tratamiento, los que abandonaron el tratamiento y aquellos que todavía continúan en tratamiento).

Todos los datos fueron registrados en una planilla de Excel que permitió preparar un banco de datos para posteriormente llevar a un programa estadístico STATA versión 14.0, se utilizó la estadística descriptiva para las frecuencias absolutas y relativas, y los test de Chi cuadrada y de Poisson fueron utilizados con un nivel de significancia de $0.05 \%$ a un nivel de confiabilidad del $95 \%$, para determinar asociación entre variables.

\section{Resultados}

Se analizaron un total de 211 historias clínicas. Los resultados obtenidos se exponen a continuación y en la tabla 1:

Se observa una prevalencia discreta del sexo femenino $(50.71 \%)$, el grupo etario de 51 a 60 años representa el $20.38 \%$, instrucción primaria el $90.52 \%$, lugar de residencia «sierra» el $76.78 \%$ y la ocupación quehaceres domésticos representa el $38.4 \%$, seguido de agricultor, con el $22.7 \%$.

En la tabla 2 se observa que de acuerdo con la CIE-10 prevalecen los códigos: C76.0 (cánceres que ocupan regiones amplias) con el $19.9 \%$ y C01-C02 (cáncer de lengua) con el 18.5\%. La región anatómica que prevalece es la cavidad bucal $(36.5 \%)$, el tratamiento recibido más prevalente es el quirúrgico $(38.9 \%)$, el tiempo de tratamiento hospitalario más frecuente es de 1 a 180 días (44.5\%). Respecto a la evolución del tratamiento, el $34.1 \%$ continuó en tratamiento y el $31.8 \%$ vivos concluyeron el tratamiento (Tabla 3).

Entre los tipos de cáncer de cabeza y cuello, de acuerdo con la $\mathrm{CIE}-10$ y las características demográficas, se puede observar que los niveles de significancia son superiores a 0.05 a un nivel de confiabilidad del $95 \%$, por lo tanto, no existe ningún tipo de asociación. Sin embargo, para el lugar de residencia el nivel de significancia es de 0.049 , existe una leve asociación con los tipos de cáncer.

En la tabla 4 se puede observa que los niveles de significancia son superiores al $0.05 \%$, a un nivel de confiabilidad del $95 \%$, por tanto, no existe asociación entre los tipos de cáncer de acuerdo con la CIE-10 y las variables clínicas del tratamiento.

En la tabla 5 se puede observar que el $13.2 \%$ de los cánceres de cabeza y cuello son de edad entre 51 y 60 años, se encuentran fuera de la cavidad bucal más en el sexo masculino (32.7\%), en pacientes con instrucción baja, en la sierra, de los cuales el $29.8 \%$ recibe varios tratamientos, con un tiempo de tratamiento hospitalario de más de 180 días, y el $43.1 \%$ no continúo el tratamiento. No existe asociación entre las variables región anatómica y variables demográficas y clínicas.

En la tabla 6 se observa la edad que no continua el tratamiento es de $51-60$ años con el $14.2 \%$, el sexo masculino con el $33.6 \%$, de la región sierra con el 
Tabla 1. Distribución de frecuencias demográficas: sexo, edad, nivel de instrucción, residencia y profesión.

Hospital de Especialidades Eugenio Espejo. 2002-2015

\begin{tabular}{|c|c|c|c|}
\hline Variables demográficas & Frecuencia & Porcentaje & Cum. \\
\hline $\begin{array}{l}\text { Sexo } \\
\text { Masculino } \\
\text { Femenino }\end{array}$ & $\begin{array}{l}104 \\
107\end{array}$ & $\begin{array}{l}49.29 \\
50.71\end{array}$ & $\begin{array}{c}49.29 \\
100\end{array}$ \\
\hline $\begin{array}{l}\text { Edad (años) } \\
11-20 \\
21-30 \\
31-40 \\
41-50 \\
51-60 \\
61-70 \\
71-80 \\
81-93\end{array}$ & $\begin{array}{l}12 \\
16 \\
25 \\
26 \\
43 \\
30 \\
31 \\
18\end{array}$ & $\begin{array}{c}5.69 \\
12.32 \\
11.85 \\
12.32 \\
20.38 \\
14.22 \\
14.69 \\
8.53\end{array}$ & $\begin{array}{r}5.69 \\
18.01 \\
29.86 \\
42.18 \\
62.56 \\
76.78 \\
91.47 \\
100\end{array}$ \\
\hline $\begin{array}{l}\text { Nivel de instrucción } \\
\text { Ninguno } \\
\text { Primaria } \\
\text { Secundaria } \\
\text { Superior }\end{array}$ & $\begin{array}{c}33 \\
112 \\
46 \\
20\end{array}$ & $\begin{array}{l}15.6 \\
53.1 \\
21.8 \\
9.48\end{array}$ & $\begin{array}{l}15.6 \\
68.7 \\
90.5 \\
100\end{array}$ \\
\hline $\begin{array}{l}\text { Residencia } \\
\text { Costa } \\
\text { Amazonía } \\
\text { Sierra }\end{array}$ & $\begin{array}{c}39 \\
10 \\
162\end{array}$ & $\begin{array}{c}18.5 \\
4.7 \\
76.78\end{array}$ & $\begin{array}{l}18.5 \\
23.2 \\
100\end{array}$ \\
\hline $\begin{array}{l}\text { Ocupación } \\
\text { Agricultor/horticultor/ } \\
\text { floricultor } \\
\text { Quehaceres domésticos } \\
\text { Estudiante } \\
\text { Comerciante } \\
\text { Área profesional } \\
\text { Artesano }\end{array}$ & $\begin{array}{l}48 \\
81 \\
25 \\
25 \\
11 \\
21\end{array}$ & $\begin{array}{c}22.7 \\
\\
38.4 \\
11.8 \\
11.8 \\
5.2 \\
10\end{array}$ & $\begin{array}{c}22.7 \\
\\
61.1 \\
73 \\
84.8 \\
90 \\
100\end{array}$ \\
\hline Total & 211 & 100.00 & \\
\hline
\end{tabular}

$51.1 \%$; con cáncer fuera de la cavidad bucal el $43.1 \%$ recibió un solo tratamiento y el $34.5 \%$ con tratamiento más de 180 días. El nivel de significancia de los tratamientos recibidos es de 0.006 , por lo tanto, existe asociación entre la evolución del tratamiento y el tratamiento recibido.

\section{Discusión}

El cáncer es un problema de salud pública, siendo los individuos con niveles socioeconómicos bajos los más vulnerables. Según Arias, et al. ${ }^{25}$, en el mundo el cáncer de cabeza y cuello ocupa el séptimo lugar de mortalidad, representando aproximadamente 375.000 muertes al año, de los cuales el $5.8 \%$ ocurren en América Latina. Este es el primer estudio de prevalencia de cáncer de cabeza y cuello hecho en el Hospital de Especialidades Eugenio Espejo del
Tabla 2. Distribución de las frecuencias relacionadas con el tipo de cáncer de cabeza y cuello, de acuerdo con la Clasificación Internacional de enfermedades (CIE-10), localización anatómica, tratamiento recibido, tiempo de tratamiento hospitalario, evolución del tratamiento. Hospital de Especialidades Eugenio Espejo. 20002-2015

\begin{tabular}{|c|c|c|c|}
\hline Diagnóstico CIE-10 & Frecuencia & Porcentaje & Cum. \\
\hline $\mathrm{COO}$ & 5 & 2.4 & 2.4 \\
\hline $\mathrm{CO1}, \mathrm{CO2}$ & 39 & 18.5 & 20.9 \\
\hline $\mathrm{CO3}$ & 11 & 5.2 & 26.1 \\
\hline C04, C05, C06.0, C06.2 & 12 & 5.7 & 31.8 \\
\hline C07, C08.0 & 34 & 16.1 & 47.9 \\
\hline C09, C10 & 21 & 10.0 & 57.8 \\
\hline C11, C310, C41.0 & 34 & 16.1 & 73.9 \\
\hline C14 & 13 & 6.2 & 80.1 \\
\hline C76.0 & 42 & 19.9 & 100.0 \\
\hline $\begin{array}{l}\text { Localización anatómica } \\
\text { Cabeza } \\
\text { Cara } \\
\text { Cavidad oral } \\
\text { Orofaringe } \\
\text { Nasofaringe } \\
\text { Glándulas salivales } \\
\text { Cuello }\end{array}$ & $\begin{array}{c}6 \\
43 \\
77 \\
21 \\
21 \\
9 \\
34 \\
21\end{array}$ & $\begin{array}{c}2.8 \\
20.4 \\
36.5 \\
10.0 \\
4.3 \\
16.1 \\
10.0\end{array}$ & $\begin{array}{c}2.8 \\
23.2 \\
59.7 \\
69.7 \\
73.9 \\
90.0 \\
100.0\end{array}$ \\
\hline $\begin{array}{l}\text { Tratamiento recibido } \\
\text { Radioterapia } \\
\text { Quimioterapia } \\
\text { Quirúrgico } \\
\text { Quimioterapia, } \\
\text { radioterapia, quirúrgica } \\
\text { Radioterapia y cirugía } \\
\text { Radioterapia y } \\
\text { quimioterapia } \\
\text { Quimioterapia y cirugía } \\
\text { Paliativo }\end{array}$ & $\begin{array}{c}2 \\
18 \\
18 \\
82 \\
33 \\
\\
21 \\
21 \\
\\
\\
33 \\
1\end{array}$ & $\begin{array}{c}0.9 \\
8.5 \\
38.9 \\
15.6 \\
\\
10.0 \\
10.0 \\
\\
15.6 \\
0.5\end{array}$ & $\begin{array}{r}0.9 \\
9.5 \\
48.3 \\
64.0 \\
\\
73.9 \\
83.9 \\
\\
99.5 \\
100.0\end{array}$ \\
\hline $\begin{array}{l}\text { Tiempo de tratamiento } \\
\text { hospitalario } \\
\text { De } 1 \text { a } 180 \text { días } \\
\text { De } 181 \text { a } 365 \text { días } \\
\text { De } 366 \text { a } 730 \text { días } \\
\text { De } 731 \text { a } 4,931 \text { días }\end{array}$ & $\begin{array}{l}94 \\
39 \\
37 \\
41\end{array}$ & $\begin{array}{l}44.5 \\
18.5 \\
17.5 \\
19.4\end{array}$ & $\begin{array}{c}44.5 \\
63.0 \\
80.6 \\
100.0\end{array}$ \\
\hline $\begin{array}{l}\text { Evolución del tratamiento } \\
\text { Continúa en tratamiento } \\
\text { Vivo, concluyó } \\
\text { tratamiento } \\
\text { Abandonó el } \\
\text { tratamiento } \\
\text { Murió durante el } \\
\text { tratamiento }\end{array}$ & $\begin{array}{l}72 \\
67 \\
54 \\
18\end{array}$ & $\begin{array}{l}34.1 \\
31.8 \\
\\
25.6 \\
\\
8.5\end{array}$ & $\begin{array}{r}34.1 \\
65.9 \\
91.5 \\
100.0\end{array}$ \\
\hline Total & 211 & 100.0 & \\
\hline
\end{tabular}

Ecuador, en donde se presentó una prevalencia discreta del sexo femenino, con el $50.71 \%$ de los casos atendidos; esta mayor incidencia tal vez ocurra debido 


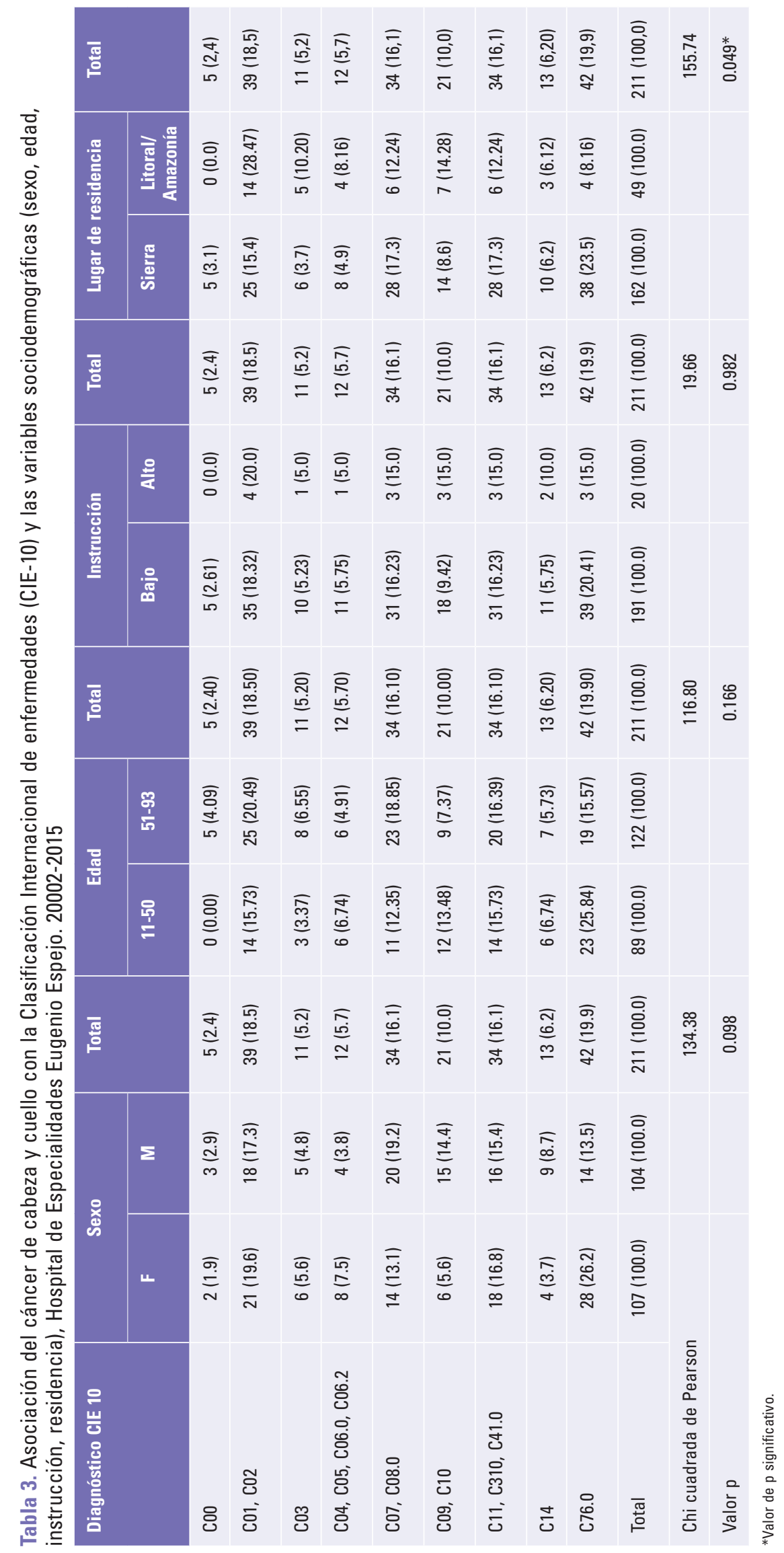


Tabla 4. Asociación del cáncer de cabeza y cuello entre la Clasificación Internacional de enfermedades (CIE-10) y las variables clínicas. Hospital de Especialidades Eugenio Espejo. 20002-2015

\begin{tabular}{|c|c|c|c|c|c|c|c|c|c|}
\hline \multirow{2}{*}{$\begin{array}{l}\text { Diagnóstico } \\
\text { CIE-10 }\end{array}$} & \multicolumn{3}{|c|}{ Tipo de tratamiento } & \multicolumn{3}{|c|}{ Tiempo de tratamiento Hosp. } & \multicolumn{3}{|c|}{ Evolución del tratamiento } \\
\hline & Único & Varios & Total & $\begin{array}{l}1-180 \\
\text { días }\end{array}$ & + 180 días & Total & $\begin{array}{l}\text { Continúan } \\
\text { en } \\
\text { tratamiento }\end{array}$ & $\begin{array}{c}\text { No } \\
\text { continúan } \\
\text { en } \\
\text { tratamiento }\end{array}$ & Total \\
\hline $\mathrm{COO}$ & $4(3.88)$ & $1(0.92)$ & $5(2.40)$ & $1(1.1)$ & $4(3.4)$ & $5(2.4)$ & $0(0.00)$ & $5(3.59)$ & $5(2,40)$ \\
\hline $\mathrm{CO1}, \mathrm{CO2}$ & $14(13.59)$ & $25(23.14)$ & $39(18.50)$ & $19(20.2)$ & $20(17.09)$ & $39(18.5)$ & $17(23.60)$ & $22(15.82)$ & $39(18,50)$ \\
\hline $\mathrm{CO3}$ & $3(2.91)$ & $8(7.40)$ & $11(5.20)$ & $5(5.3)$ & $6(5.12)$ & $11(5.2)$ & $5(6.90)$ & $6(4.31)$ & $11(5,20)$ \\
\hline $\begin{array}{l}\text { CO4, C05, } \\
\text { C06.0, C06.2 }\end{array}$ & $4(3.88)$ & $8(7.40)$ & $12(5.70)$ & $5(5.3)$ & 7 (5.98) & $12(5.7)$ & $6(8.30)$ & $6(4.31)$ & $12(5,70)$ \\
\hline C07, C08.0 & 18 (17.47) & $16(14.81)$ & $34(16.10)$ & $13(13.8)$ & $21(17.94)$ & $34(16.1)$ & $11(15.30)$ & $23(16.54)$ & $34(16,10)$ \\
\hline C09, C10 & $8(7.76)$ & $13(12.03)$ & $21(10.00)$ & $6(6.4)$ & $15(12.82)$ & $21(10.0)$ & $9(12.50)$ & $12(8.63)$ & $21(10,00)$ \\
\hline $\begin{array}{l}\text { C11, C310, } \\
\text { C41.0 }\end{array}$ & $17(16.50)$ & 17 (15.74) & $34(16.10)$ & $20(21.3)$ & $14(11.96)$ & $34(16.1)$ & $9(12.50)$ & 25 (17.98) & $34(16,10)$ \\
\hline C14 & $8(7.76)$ & $5(4.62)$ & $13(6.20)$ & $8(8.5)$ & $5(4.27)$ & $13(6.2)$ & $2(2.80)$ & $11(7.91)$ & $13(6,20)$ \\
\hline C76.0 & $27(26.21)$ & $15(13.88)$ & $42(19.90)$ & $17(18.1)$ & $25(21.36)$ & 42 (19.9) & $13(18.10)$ & $29(20.86)$ & $42(19,90)$ \\
\hline Total & $103(100)$ & $108(100)$ & $211(100.0)$ & $94(100.0)$ & $117(100.0)$ & $211(100.0)$ & $72(100.0)$ & $139(100.0)$ & $211(100,0)$ \\
\hline \multicolumn{2}{|c|}{ Chi cuadrada de Pearson } & & 138.27 & & & 88.63 & & & 99.83 \\
\hline \multicolumn{2}{|l|}{ Valor $p$} & & 0.086 & & & 0.371 & & & 0.266 \\
\hline
\end{tabular}

a cambios que han surgido en los últimos años de comportamiento y calidad de vida de las mujeres, como la mala alimentación, aumento en el consumo de tabaco y alcohol y ciertos comportamientos sexuales a edad temprana, y en el caso de las amas de casa podría ser por el uso constante de productos químicos para limpieza y desinfección. La OMS y estudios realizados por Pereira, et al. ${ }^{26}$, Oreggioni, et al. ${ }^{7}$ y Posada, et al. ${ }^{23}$ mencionan que existe un predominio del sexo masculino.

Varios estudios han determinado que la edad media de los pacientes afectados con cáncer de cabeza y cuello es de $60 \pm 5$ años y la incidencia aumenta con la edad próxima a los 40 años. En este estudio el grupo etario fue de 11 a 93 años, con una media de $55 \pm 5$ años, datos que concuerdan con otros estudios realizados por Vinitzky, et al. ${ }^{27}$ (edad promedio de 60.5 años), Oreggioni, et al. ${ }^{7}$ (media de $55 \pm 12$ ) y Posada, et al. ${ }^{23}$ (63.5 años). Alan, et al. ${ }^{28}$, en su estudio realizado en la India, presentan mayor porcentaje en el grupo etario de 51 a 60 años.

En estudios realizados sobre cáncer de cabeza y cuello se ha demostrado que los pacientes con bajos niveles de educación y económicos son los más vulnerables para el desarrollo del cáncer. De acuerdo con los niveles de escolaridad, Moraes, et al. ${ }^{29}$, en Brasil, encontraron nivel «ninguno, primaria y secundaria» con el $97.1 \%$, lo que presenta cierta similitud con nuestro estudio, en el cual se observó el $90.52 \%$ de nivel «ninguno, primaria y secundaria». Estos datos son consistentes con diferentes estudios y puede ser que este alto porcentaje esté relacionado con el bajo conocimiento por parte de los pacientes sobre la misma enfermedad, los factores de riesgo, la sintomatología y los cuidados que deben tener para prevenir el cáncer, tornándose un grupo vulnerable con acceso limitado para un diagnóstico precoz.

Es importante recalcar que el cáncer se manifiesta en diferentes regiones anatómicas y puede estar relacionado con diferentes estilos de vida, raza, sexo, genética y también ser influenciado por las condiciones geográficas y ambientales de cada región. El Ecuador es un país con gran diversidad geográfica, social, cultural, ambiental y, por su localización, es un territorio que queda entre ambos hemisferios. El clima es variado, tiene acceso al Océano Pacífico, al Amazonas, a las Islas Galápagos y a la Cordillera de los Andes, 
Tabla 5. Asociación del cáncer de cabeza y cuello entre la región anatómica y las variables demográficas y clínicas. Hospital de Especialidades Eugenio Espejo. 20002-2015

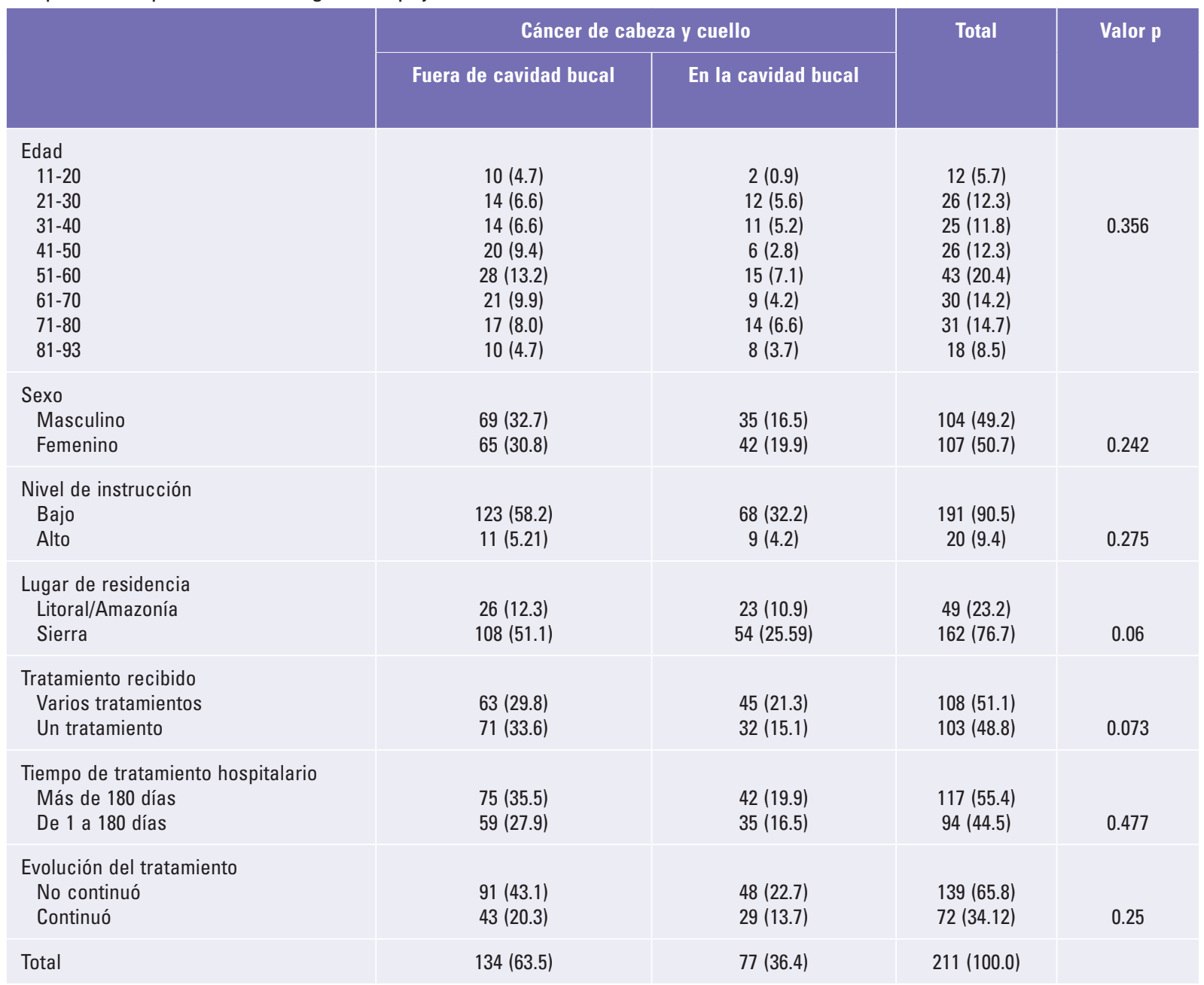

dando origen a la formación de cuatro regiones, que son litoral o costa, sierra, Amazonía y región insular. Para nuestro estudio se consideraron las regiones: sierra, costa y oriente; se dicotomizó de acuerdo con ciertas características, en la sierra existen montañas frías y altas en relación con la costa y en la Amazonía hay áreas de clima tropical y caliente. Se obtuvo un $76.78 \%$ de los pacientes atendidos de la sierra. Esta mayor prevalencia en la sierra puede darse tal vez porque el Hospital de Especialidades Eugenio Espejo es estatal y está ubicado en la región sierra (Quito, Ecuador), siendo más próximo y accesible para las personas que viven cerca de este lugar, sin dejar de ser un centro de referencia nacional.

Diferentes estudios relatan que algunos tipos de ocupación, oficios o actividades pueden estar asociados a ciertos tipos de cáncer. Alvarenga, et al. ${ }^{30}$, en Brasil, en un estudio epidemiológico del cáncer de cabeza y cuello de acuerdo con la ocupación determinaron que prevalece más en pacientes agricultores y dedicados a quehaceres domésticos, dándose similar situación con el presente estudio, en donde el $22.7 \%$ son agricultores y el $38.4 \%$ se dedica a los quehaceres domésticos. Estos grupos poblacionales pueden ser más afectados tal vez debido a la falta de conocimiento de la enfermedad y a los niveles bajos de escolaridad; y el cáncer en sus estadios iniciales es asintomático, razón por la cual muchas personas reciben su diagnóstico y tratamiento cuando el cáncer está en estadios avanzados. Los agricultores son personas que el mayor tiempo están expuestas o relacionadas con la tierra, el sol y ciertos cambios ambientales. Los dedicados a quehaceres domésticos son personas que laboran en casa, generalmente son mujeres y para la 
Tabla 6. Asociación del cáncer de cabeza y cuello entre la evolución del tratamiento y las variables demográficas y clínicas. Hospital de Especialidades Eugenio Espejo. 20002-2015

\begin{tabular}{|c|c|c|c|c|}
\hline & \multicolumn{2}{|c|}{ Cáncer de cabeza y cuello } & \multirow{2}{*}{$\begin{array}{c}\text { Total } \\
(\%)\end{array}$} & \multirow[t]{2}{*}{$\mathbf{p}$} \\
\hline & No continuó tratamiento & $\begin{array}{l}\text { Continuó } \\
\text { tratamiento }\end{array}$ & & \\
\hline $\begin{array}{c}\text { Edad } \\
11-20 \\
21-30 \\
31-40 \\
41-50 \\
51-60 \\
61-70 \\
71-80 \\
81-93\end{array}$ & $\begin{array}{c}10(4.7) \\
15(7.1) \\
15(7.1) \\
16(7.5) \\
30(14.2) \\
19(9.0 \\
21(9.9) \\
13(6.1)\end{array}$ & $\begin{array}{c}2(0.9) \\
11(5.2) \\
10(4.7) \\
10(4.7) \\
13(6.1) \\
11(5.2) \\
10(4.7) \\
5(2.3)\end{array}$ & $\begin{array}{c}12(5.7) \\
26(12.3) \\
25(11.8) \\
26(12.3) \\
43(20.4) \\
30(14.2) \\
31(14.7) \\
18(8.5)\end{array}$ & 0.823 \\
\hline $\begin{array}{l}\text { Sexo } \\
\text { Masculino } \\
\text { Femenino }\end{array}$ & $\begin{array}{l}71(33.6) \\
68(32.2)\end{array}$ & $\begin{array}{l}33(15.6) \\
39(18.4)\end{array}$ & $\begin{array}{l}104(49.2) \\
107(50.7)\end{array}$ & 0.282 \\
\hline $\begin{array}{l}\text { Instrucción } \\
\text { Bajo } \\
\text { Alto }\end{array}$ & $\begin{array}{c}124(58.7) \\
15(7.1)\end{array}$ & $\begin{array}{c}67(31.7) \\
5(2.3)\end{array}$ & $\begin{array}{c}191(90.5) \\
20(9.4)\end{array}$ & 0.26 \\
\hline $\begin{array}{l}\text { Lugar de residencia } \\
\text { Litoral/Amazonía } \\
\text { Sierra }\end{array}$ & $\begin{array}{c}31(14.6) \\
108(51.1)\end{array}$ & $\begin{array}{c}18(8.5) \\
54(25.5)\end{array}$ & $\begin{array}{l}49(23.22) \\
162(76.7)\end{array}$ & 0.391 \\
\hline $\begin{array}{l}\text { Región anatómica } \\
\text { Otras regiones } \\
\text { Cavidad bucal }\end{array}$ & $\begin{array}{l}91(43.1) \\
48(22.7)\end{array}$ & $\begin{array}{l}43(20.3) \\
29(13.7)\end{array}$ & $\begin{array}{l}134(63.5) \\
77(36.4)\end{array}$ & 0.25 \\
\hline $\begin{array}{l}\text { Tratamiento recibido } \\
\text { Varios tratamientos } \\
\text { Un tratamiento }\end{array}$ & $\begin{array}{l}62(29.3) \\
77(36.4)\end{array}$ & $\begin{array}{l}46(21.8) \\
26(12.3)\end{array}$ & $\begin{array}{l}108(51.1) \\
103(48.8)\end{array}$ & 0.006 \\
\hline $\begin{array}{l}\text { Tiempo de tratamiento hospitalario } \\
\text { Más de } 180 \text { días } \\
\text { De } 1 \text { a } 180 \text { días }\end{array}$ & $\begin{array}{l}73(34.5) \\
66(31.2)\end{array}$ & $\begin{array}{l}44(20.8) \\
28(13.2)\end{array}$ & $\begin{array}{c}117(55.4) \\
94(44.5)\end{array}$ & 0.148 \\
\hline Total & $139(65.8)$ & $72(34.1)$ & $211(100.0)$ & \\
\hline
\end{tabular}

limpieza y desinfección de sus casas están expuestas constantemente a ciertas sustancias que pueden o no generar humo, polvo y productos químicos, como los desinfectantes, en especial el cloro en altas concentraciones.

Para identificar el tipo de cáncer en nuestro estudio se utilizó la CIE-10, códigos utilizados en el hospital Eugenio Espejo: el 19.9\% de los pacientes con cáncer de cabeza y cuello corresponde al código C76.0 (este código se utiliza para designar las neoplasias que no poseen una localización específica o por ser cánceres que ocupan grandes extensiones anatómicas), seguido tenemos el cáncer en la lengua (C01-C02) con el $18.5 \%$ y de las glándulas salivales (C07-C08.0), que representaron el $16.1 \%$ de los casos, siendo la localización anatómica más afectada por el cáncer la cavidad bucal con el $36.5 \%$, glándulas salivales el $16.1 \%$ y la orofaringe con el 10\%. Datos similares se encontraron en los estudios realizados por Ordóñez, et al. ${ }^{31}$, Álvarez Arias, et al. ${ }^{25}$ y Oreggioni, et al. ${ }^{7}$.

Dependiendo del tipo y estadio del cáncer los tratamientos podrían ser únicos o combinadas, como mencionan Posada, et al. ${ }^{23}$ y Vargas, et al. ${ }^{9}$, existiendo cierta similitud con nuestro estudio, en donde los tratamientos combinados fueron representados con el $51.18 \%$ y el quirúrgico como único tratamiento con el $48.82 \%$.

El $44.5 \%$ tuvo un tiempo de tratamiento hospitalario de 1 a 180 días, tiempo que fue considerado desde la fecha del primer día que el paciente acude para el diagnóstico y tratamiento hasta la última fecha registrada en la historia clínica, existiendo pacientes que permanecieron bajo tratamiento más de dos años. El $31.8 \%$ de pacientes terminó el 
tratamiento, el $25.6 \%$ abandonaron el tratamiento, el $8.5 \%$ murió durante el tratamiento y el $34.1 \%$ continúan en tratamiento. Es importante recalcar que para este estudio los datos fueron tomados hasta el 31 de diciembre del 2015; se puede observar que existe mayor afluencia de pacientes atendidos con cáncer de cabeza y cuello durante los años 2013, 2014 y durante el año 2015, por lo tanto, muchos continuaban en tratamiento.

Este estudio epidemiológico ha sido muy importante, ya que nos permitió tener ciertos datos estadísticos y con ellos saber cuáles son los grupos más vulnerables, para poder implementar acciones de educación y cuidado que las personas deben tener antes de que se desencadene $o$ avance esta enfermedad. También, al ser un problema de salud pública, es necesario trabajar en conjunto con instituciones privadas para adoptar medidas necesarias para prevenir, educar y direccionar a las personas al lugar adecuado para los tratamientos y cuidados necesarios.

\section{Agradecimientos}

Al Hospital de Especialidades Eugenio Espejo, especialmente al Líder del Servicio de Oncología Dr. Ahmad Wali Mushtaq Wali.

\section{Financiamiento}

Los autores no recibieron patrocinio para llevar a cabo este artículo.

\section{Conflicto de intereses}

Los autores declaran no tener conflicto de intereses alguno.

\section{Responsabilidades éticas}

Protección de personas y animales. Los autores declaran que para esta investigación no se han realizado experimentos en seres humanos ni en animales.

Confidencialidad de los datos. Los autores declaran que han seguido los protocolos de su centro de trabajo sobre la publicación de datos de pacientes.

Derecho a la privacidad y consentimiento informado. Los autores declaran que en este artículo no aparecen datos de pacientes.

\section{Bibliografía}

1. Granados García M, Herrera Gómez Á. Manual de oncología. Procedimientos médicos quirúrgicos. Cuarta Ed. México: McGraw-Hill; 2010. pp. $1-9$.

2. Ariosa Argüelles C, González F, Rodríguez G, Rodríguez J. Cáncer bucal. Estudio de cinco años. Revista Médica Electrónica [Internet]. 2006;28 (6). Disponible en: http://www.revmedicaelectronica.sld.cu/index. $\mathrm{php} / \mathrm{rme} / \mathrm{article} / \mathrm{view} / 349$

3. Moctezuma-Bravo GS, Díaz de León-Medina R, Rodríguez-Quilantan FJ, Moctezuma-Dávila M. Cáncer oral en un hospital general de zona del Instituto Mexicano del Seguro Social en México, (1988-2005). Gac Mex Oncol. 2015;14(6):323-8.

4. Navarro Expósito F, López González JL, Álvarez-Mon Soto M. Cáncer de cabeza y cuello. Med - Programa Form Médica Contin Acreditado [Internet]. 2017;12(31):1833-48. Disponible en: http://linkinghub.elsevier. com/retrieve/pii/S0304541217300872

5. Gallegos-Hernández JF. Cáncer de cabeza y cuello. Gac Mex Oncol. 2015;14(1):1-2.

6. Gallegos Hernández JF, Ortiz Maldonado AL, Rojas Orellana S, Flores Díaz R, Espinoza Velazco A, Minauro Muñoz GG. Factores pronóstico en cáncer de boca. Acta Médica Grupo Ángeles. 2010;8(2):88-94.

7. Oreggioni Aldama L, Ortíz L, Joy L, Moringo M. Desnutrición a partir de la valoración global subjetiva generada por el paciente (VGS-GP) en pacientes con cáncer de cabeza y cuello. Mem Inst Investig Cienc Salud. 2016;14(1):86-93.

8. Coca Granado MR, Jiménez Rodríguez Y. El cáncer de cabeza y cuello: un problema social. Acta Med Cent. 2015;9(4):112-6.

9. Vargas-Soto O, Molina-Fechero M, Castañeda-Castaneira E, Bolonga-Molina R, Carreón-Burciaga R, González-González R. Carcinomas de cabeza y cuello, experiencia de un Centro Oncológico del Estado de Durango, México. Rev ADM. 2016;73(4):190-6.

10. Ferlay J, Soerjomataram I, Ervik M, Dikshit R, Eser S, Mathers C, et al. GLOBOCAN 2012 v1.0. Cancer Incidence and Mortality Worldwide: IARC CancerBase. No. 11 [Internet]. Lyon, France: International Agency for Research on Cancer; 2013. Disponible en: http://globocan.iarc.fr

11. Biazevic MGH, Castellanos RA, Antunes JLF, Michel-Crosato E, Crosato EM. Tendências de mortalidade por câncer de boca e orofaringe no Município de São Paulo, Brasil, 1980/2002. Cad Saúde Pública, Rio Janeiro [Internet]. 2006;22(10):2105-14. Disponible en: http://www.scielo. br/scielo.php?script=sci_arttext\&pid=S0102-311X2006001000016\&nr$\mathrm{m}=$ iso

12. Koontongkaew S. The tumor microenvironment contribution to development, growth, invasion and metastasis of head and neck squamous cell carcinomas. J Cancer. 2013;4(1):66-83.

13. Sepiashvili L, Hui A, Ignatchenko V, Shi W, Su S, Xu W, et al. Potentially novel candidate biomarkers for head and neck squamous cell carcinoma identified using an integrated cell line-based discovery strategy. Mol Cell Proteomics. 2012;11(11):1404-15.

14. Abreu de Aquino R, Lopes M, Ximenes de Menezes C, Rodrígues M. Alterações fonoaudiológicas e acesso ao fonoaudiólogo nos casos de óbito por câncer de lábio, cavidade oral e orofaringe: um estudo retrospectivo. Rev CEFAC [Internet]. 2016;18(3):737-45. Disponible en: http:// www.scielo.br/scielo.php? script $=$ sci_arttext\&pid $=S 1516$ 18462016000300737\&lng=pt\&tlng=pt

15. Cardemil MF. Epidemiología del carcinoma escamoso de cabeza y cuello. Rev Chil Cir. 2014;66(6):614-20.

16. International Agency for Research on cancer. World cancer report 2014 [Internet]. International Agency for Research on cancer, World Health Organization; 2014. Disponible en: https://www.who.int/cancer/publications/WRC 2014/en/

17. Biazevic MGH, Antunes JLF, Togni J, de Andrade FP, de Carvalho MB, Wünsch-Filho V. Immediate impact of primary surgery on health-related quality of life of hospitalized patients with oral and oropharyngeal cancer. J Oral Maxillofac Surg. 2008;66(7):1343-50.

18. Pinaki B, Brockton NT, Dort JC. Head and neck cancer: From anatomy to biology. Int J Cancer. 2013;133(9):2013-23.

19. Parkin DM, Pisani P, Ferlay J. Global Cancer Statistics. CA Cancer J Clin. 1999;49(1):33-64

20. Torre L, Bray F, Siegel RL, Ferlay J, Lortet-Tieulent J, Jemal A. Global Cancer Statistics, 2012. CA Cancer J Clin. 2015;65(2):87-108.

21. Kocaelli H, Apaydin A, Aydil B, Ayhan M, Karadeniz A, Ozel S, et al. Evaluation of potential salivary acetaldehyde production from ethanol in oral cancer patients and healthy subjects. Hippokratia. 2014;18: 269-74.

22. Rocha Buelvas A. Cáncer oral: El papel del odontólogo en la detección temprana y control. Rev Fac Odontol Univ Antioq. 2009;21(1):112-21.

23. Posada-López A, Palacio M, Salas C, Álvarez E, Grisales H. Survival rate of oral squamous cell carcinoma patients treated for the first time in cancer centers between 2000 and 2011, Medellín-Colombia. Rev Fac Odontol Univ Antioq. 2016;27(2):245-61 
24. Sociedad de Lucha Contra el Cáncer QE. Registro Nacional De Tumores/ National Cancer Registry. Epidemiología del cáncer en Quito 2006-2010 [Internet]. Quito, Ecuador: Sociedad de Lucha contra el Cáncer QE; 2014 Disponible en: http://www.estadisticas.med.ec/Publicaciones/PUBLICACION-QU-2006-2010.pdf

25. Arias DÁ, Estefan AM, Borche G, Albora RD, Sande VR, Cuello M. Cáncer de cabeza y cuello en Uruguay. Análisis de sobrevida en dos centros de referencia. 2018:34(1):21-8. Disponível em: http://dx.doi.org/10.29193/rmu.34.1.2

26. Pereira IF, Reimar V, de Souza Noronha A, Drummond M, Mara T, Amaral P, et al. Neoplasias malignas em região de cabeça e pescoço: perfil dos pacientes atendidos na UFMG. Rev Cubana Estomatol. 2016:53(4)

27. Vinitzky Brener I, Ibáñez Mancera NG, Eljure Eljure E, Bravo FA. Retraso en el diagnóstico de cáncer en cavidad bucal y anexos como factor clave para el pronóstico. Rev ADM. 2014;71(4):188-91.
28. Alam M, Siddiqui S, Perween R. Epidemiological profile of head and neck cancer patients in Western Uttar Pradesh and analysis of distributions of risk factors in relation to site of tumor. $\mathrm{J}$ Cancer Res Ther. 2017:13(3):430-5

29. Costa de Moraes R, Dias F, Marcelo da Silva C, Guimäraes Fischer R. Association between chronic periodontitis and oral/oropharyngeal cancer. Braz Dent. 2016;27(3):261-6.

30. Alvarenga L, Ruiz M. Avaliação epidemiológica de pacientes com câncer de cabeça e pescoço em um hospital universitário do noroeste do estado de São Paulo. Braz J Otorhinolaryngol. 2008; 74(1):68-73.

31. Ordóñez D, Aragón N, García LS, Collazos P, Bravo LE. Cáncer oral en Santiago de Cali, Colombia: análisis poblacional de la tendencia de incidencia y mortalidad. Salud Publica Mex. 2014;56(5):465-72. 